\title{
ZUM EPINALGLOSSAR.
}

Krankheit hatte mich leider arg in rückstand mit meiner arbeit gebracht, so dafs ich aufser stande war, im letzten hefte die fortsetzung meiner vergleichung mit der hs. zu geben. In der zwischenzeit hat mir prof. Hecht den vorschlag gemacht, eine neuausgabe des Epinaler glossars für die GreinWülkersche bibliothek der angelsächsischen prosa $z \mathbf{z}$ besorgen und meine forschungen darin za verwerten. Ich bin daranf um so lieber eingegangen, als der plan der neuen ausgabe ein zuverlässiges facsimile einschlofs, das mit der transliteration den ersten teil bilden und besonders verkänflich sein soll. Wenn die umstände günstig sind, mag es noch diesen herbst erscheinen. Der leser wird sich dann aus eigner anschaung ein urteil darüber bilden können, in wie weit Sweet's ausgabe der hs. gerecht wird. Mit zustimmung prof. Einenkels verzichte ich daher hier auf weitere vorfuhrung der ergebnisse meiner vergleichung nnd beschränke mich darauf, einiges von der einleitung berichtigen, die ich im vorletzten hefte veröffentlicht habe. Erneute einsicht des eintrages anf dem ersten blatte der Sermones hat mich zu der überzengung gebracht, dafs zu lesen ist, wie folgt:

Ite lib ẽ de majoz echa ezo johef fect eũ relizarj

Der sich da nennende Johannes hat also den Codex nicht geschrieben, sondern ihn einbinden lassen. Die hs. der Sermones Augustini war demnach einst ein selbständiger band, ehe sie im 18. jahrhundert mit dem Glossare zusammen vereinigt wurde. Der einband mufs ziemlich wertvoll gewesen sein; sonst wäre er wohl nicht entfernt und bei der inventarisierung ein zusammenbinden mit einer andern hs. nötig geworden. Was den eintrag auf der ersten seite des glossars anbelangt, so ermöglicht mir meine photographie, jetzt folgendes mit einer gewissen sicherheit als die wahrscheinliche lesung zu bezeichnen: Lexicon Anglosaxonicum vetus. vide, si placet, Hickesij thesaurum.

Nachschrift: Ich glaube den interessen der anglistik zu dienen, wenn ich wenigstens einige allgemeine bemerkungen mache, die sich mir aus dem vergleiche von Sweet's ausgabe mit der hs. ergeben haben und einige beispiele anführe zur erhärtung des gesagten. Im vorbemerk lehnt Sweet ausdrück- 
lich die verantwortung für die facsimile-reproduktionen seiner ausgabe ab, trägt aber kein bedenken zu sagen, dafs er glaubt 'that on the whole they represent the Ms. accurately, although, as will be easily seen, they fail in reproducing its less distinct portions'. Demgegenüber muls ich sagen, dals auch in den 'distinct portions' sein facsimile derjenigen treue ermangelt, die für zuverlässigkeit absolut notwendig ist, und durch sein transcript hat er dem mangel kaum abgeholfen. Laut p. XIV der introduktion 'the transliteration which accompanies the facsimile is quite independent of it, having been made from the Ms. itself'. Über diese transliteration sagt Goetz in der einleitung zum Corpus Glossariorum Latinorum, vol. V, p. XXVII, folgendes: 'Cuius transcriptionem cum scirem non immerito a doctis vituperatam esse, ubicumque fieri potuit exemplum phototypice expressum sequi malui.' Dazu fügt er die anmerkung: 'Meo errore factum est, ut p. 380, 43 in apparatu sperbr poneretur pro eo quod in codice est sperpi'. Er hätte erläuternd bemerken können, dafs dieser sein irrtum auf das sperbr zurückgeht, das Sweet in den OET. unter no. 769 der Epinalglossen druckt, obwohl er im transcript richtig hat sperwr. Diese unstimmigkeit zwischen Sweet's transcript und OET. beruht darauf, dafs er eine ganz falsche vorstellung von dem gebrauche der $\mathfrak{b}$ - und p-runen beim Epinalschreiber hat. Die fehler, die dieser beim abschreiben gemacht hat, weisen deutlich darauf hin, dafs er in seiner vorlage die beiden runen fand, er sie aber nicht immer auseinander zu halten vermochte, sei es wegen undeutlichkeit der vorlage, sei es wegen eigner unsicherheit über ihre bedeutung. Jedenfalls geht es nicht an, diese unsicherheit so zu erklären, wie Sweet p. XIII a der einleitung tut und im transcript p. 14 F 16 milctp zu drucken, wo facsimile $=h s$. deutlich die $p$-rune zeigen. Seine unsicherheit über die bedeutung der beiden runen bekundet ja der schreiber noch fernerhin dadurch, dals er gelegentlich auch $\mathfrak{p}$ für $\mathfrak{p}$ seiner vorlage setzt; so schreibt er fol. $1 v 1^{28}$ alga paar und korrigiert dann $\mathfrak{p}$ durch übergesetztes $\mathfrak{u}$, eine tatsache, die Sweet durch den druck im transcript p. 2 B 28 uaar vollständig verwischt. Erst in den Notes (sollte heifsen Corrections) zu ende der facsimileausgabe gibt er eine berichtigung. Diese berichtigungen des transcripts sind aber keineswegs vollständig oder immer genau. Einige weitere berich- 
tigungen enthält ein manchen exemplaren beigelegter zettel, der vom 22. April 1885 datiert ist. Ich habe ihn zuerst im exemplar der hiesigen universitätsbibliothek entdeckt. Auch Goetz kann von seinem vorhandensein keine ahnung gehabt haben; denn er gibt z. b. die falschen angaben des transcripts, p. 28 A 20 uestibulm für uestibulum, p. $28 \mathrm{C} 14$ ueria für ueneria, p. 28 C 34 undecu für undecūque als lesungen des Epinal im kritischen apparate zum Erfurt an, obwohl diese versehen auf jenem zettel berichtigt sind.

Angesichts der ungenauigkeit, die dem transcripte Sweet's an sich anhaftet und die nur teilweise durch die Notes und jenen zettel verbessert ist, wird der leser naturgemäls sich an die photolithographie halten wollen, soweit wenigstens ihr zustand eine kontrolle des transcriptes gestattet. Aber selbst in den nicht verschmierten stellen ist man nie sicher, ob der photolithograph getreu die hs. wiedergibt. So hatte ich mir vor jahren, ehe ich die hs. selbst zur einsicht bekam, folgendes als merkwürdige abweichungen des transcripts vom facsimile notiert: p. 2 D 36 staefnęndra. Nach dem facsimile hätte die hs. staefjnęndra, und zwar wäre das $j$ nachträglich hineinkorrigiert. Nichts von einem solchen $j$ aber findet sich in der hs. Ebensowenig steht in der hs. das zeichen' über dem m des Lemma alternantium, von dem das facsimile anscheinende urkunde gibt. Transcript p. 9 A 36 lesen wir uncysti $[g]$. $\mathrm{Da}$ das facsimile nur uncysti zeigt, so könnte man annehmen, das eingeklammerte $g$ beruhe auf ungenauigkeit; aber die hs. hat wirklich uncysti. Transcript p. 18 B 26 lesen wir gimengidlice. Nach dem facsimile sieht es aus, als ob ursprüngliches gimengiulice (mit offenem a) zu gimengidlice korrigiert wäre. Das ms. hat klar und deutlich zımenzıd lıce. Die tatsache eines zwischenraumes zwischen zimenzıd und tıce wird also ganz verwischt und ein falscher eindruck betreffs $\partial$ hervorgerufen. Umgekehrt bietet das facsimile das richtige, während das direkt vom ms. gemachte transcript falsch berichtet: p. $17 \mathrm{~F} 34$ treule:snis (wozu die bemerkung in den Notes, dais nach $e$ ein $u$ ausradiert sei). Das facsimile zeigt klares treuleusnis, und so steht auch in der hs.; das $u$ nach $e$ erscheint ein wenig blasser, aber von rasur ist nicht die spur. Nicht ganz klar kommt im facsimile zum ausdruck, dals das 
e nach 0 von soęrendi unterpunktiert ist, um so mehr möchte ich hier darauf aufmerksam machen, weil das transcript von diesen tilgungspunkten keine kenntnis nimmt; so bietet es z. b. p. 18 A 39 pro[u]ectae, und erst die Notes klären darüber auf, daIs $u$ über unterpunktiertem $f$ steht, wie das facsimile zeigt. Im anschlusse daran sei auch der bericht des transcripts über die nächste glosse richtig gestellt. Hier wird p. $18 \mathrm{C} 1$ gedruckt prouetae und in den Notes berichtigend bemerkt, dass $u$ über unterpunktiertem $f$ stehe. Wir müssen noch etwas weiter gehen und sagen, dals die hs. proffecte habe, wie selbst aus dem hier verschmierten facsimile zu erkennen ist. Auf unstimmigkeiten zwischen dem transcript des facsimiles und dem drucke der Epinalglosse in den OET. habe ich schon hingewiesen. Hier sei noch ein frappanter fall angeführt. OET. p. 102 lesen wir unter nr. 1034 tuta *onsorg-orsorg, als ob der Epinal hier vom Erfurt abwiche und fälschlich $n$ für $r$ biete. Facsimile und transcript p. 27 B 38 bieten richtig orsorg. Diesen fehler der OET. pflanzt weiter und verschärft W. Lehmann in seiner studie über das präfix uz- (Kieler Studien z. engl. Phil. H. 3, p. 52, anm. 1), indem er die (falsche) lesung onsorg dem Corpusglossar zuschiebt und darauf gar noch theorien aufbaut. Demgegenüber sei nachdrücklich hervorgehoben, dals Epinal-Erfurt-Corpus übereinstimmend orsorg haben. Bei dieser gelegenheit sei auch darauf hingewiesen, dals anmerkung 1 zu p. 53 von Lehmanns schrift: 'Die Sweetsche lesart von Corp. gl. = Epin.- Erf. 340 devia callis = horuaeg stiig $=$ horig $:$ horh, hor $=$ ahd. horawig $:$ horo ist, wie bekannt, nicht zu rechtfertigen', so lauten sollte: 'Sweets deutung von horuaeg (horweg) in der Ep.-Erf. glosse $340=$ Corp. 651, deuia callis horuaeg stiig (horweg stig) als 'muddy' ist nicht zu rechtfertigen, wie Schlutter nachgewiesen hat.'

Ich benutze die gelegenheit zum schlusse, ein wort über die Leidenglosse tyrfhaga 'fossorium' anzufügen. Ich tue dies aus anlals der $z$ weiten auflage von Holthausens ausgabe der Elene. In der ersten auflage lauten vv. 828-33 im anschlusse an die hs. so:

Ongan pa wilfægen æfter pam wuldres treo elnes ánhydig eorõan delfan

830 under turfhagan, [0ð-] pæt he on xx 
fotmælū feor funde behelede

under neolu niðer næsse gehydde

in peostorcofan -

In der zweiten auflage wird eine umstellung von under turfhagan vorgenommen und das fälschlich eingefügte oð- aufgegeben, so dals der vers nun lautet:

pæt he on $\mathrm{xx}[\mathrm{um}]$ under turfhagan .

Gegen diese umstellung habe ich zweierlei einzuwendep: einmal entfernt sie sich zu sehr von dem, was die hs. bietet, und zweitens beruht sie auf der erst noch zu beweisenden annahme, dals der dichter mit turfhaga den 'torfhag' = rasen bezeichnen wollte. An sich wäre nichts gegen die möglichkeit dieser annahme einzuwenden. Dagegen spricht, dals ein turfhaga 'rasen' nur an dieser stelle zu erweisen wäre, wir aber ein tyrfhaga 'ligo, fossorium' aus dem Leidener glossar und seiner sippe kennen, das trefflich zn dem texte der Acts Sanctorum palst, der der stelle zu grunde liegt. Es ist bekannt, wie eng der dichter sich an diesen text sonst angeschlossen hat. Wenn es nun in den Acta Sanctorum heifst: accipiens fossorium praecinxit se viriliter et coepit fodere, und wir damit vergleichen des dichters Ongan $b a$ wilfogen after bam wuldres treo elnes anhydig eordan delfan under turfhagan, so lälst sich kaum der gedanke abweisen, dals turfhagan der instrumentalis von turfhaga = tyrfhaga 'fossorium' ist und das accipiens fossorium der vorlage wiedergibt. Wenn irgend eine umstellung in unserm texte des gedichtes vorzunehmen ist, so dürfte es nur die sein, dals delfan und under ihre plätze vertauschen, also:

Ongan pa wilfaegen after pam wuldres treo elnes ánhydig eorðan under delfan turfhagan.

Dem abschreiber mag turf (tyrf) haga 'fossorium' nicht geläufig gewesen sein und das die umstellung veranlaIst haben, indem er wirklich an 'torfhag' denkend sich so das wort zurecht legte.

Basel (Schweiz).

Otтo B. Schlotther. 